\title{
SYNTHESIS OF A NOVEL LOW-COMPONENT PROGRAMMABLE SINUSOIDAL OSCILLATOR
}

\author{
MUHAMMAD TAHER ABUELMA'ATTI* and SA'AD MUHAMMAD AL-SHAHRANI \\ King Fahd University of Petroleum and Minerals, Box 203, Dhahran 31261, Saudi Arabia
}

(Received 30 May 2002; In final form 3 July 2002)

\begin{abstract}
A novel circuit is presented for realizing a sinusoidal oscillator. The proposed circuit, developed through a true synthesis approach, uses one current-feedback operational amplifier (CFOA), one operational transconductance amplifier (OTA), three grounded capacitors and one floating resistor. The proposed circuit enjoys several attractive features. Practical results, in excellent agreement with the presented theory, are included.
\end{abstract}

Keywords: Sinusoidal oscillators; Current feedback operational amplifiers; Operational Transconductance Amplifiers (OTA)

\section{INTRODUCTION}

Sinusoidal oscillators are widely used in instrumentation, measurement, communication and control systems. The literature contains a huge number of oscillator designs and analysis based on different principles and using operational amplifiers (OAs), operational transconductance amplifiers (OTAs), current-conveyors (CCs), current-feedback amplifiers (CFOAs) and four-terminal floating nullors (FTFNs). Of particular interest here are voltage-mode grounded-capacitors, single-resistance-controlled oscillators using two active elements and a number of grounded and/or floating resistors [1-20]. A critical study shows that these circuits suffer from one or more of the following drawbacks:

1. The lack of a synthesis approach which makes possible the evolution of the oscillator circuits $[1,4-6,9-17,19,20]$. This is because most of these circuits were discovered either by accident or with the help of intuition and various hit-and-miss techniques. In fact, even the approach used in developing the circuits reported in Refs. [7, 8, 18] is more an analytical approach rather than a synthesis approach as it relies upon the formulation of a general structure with several nodes from which various oscillator circuits can be obtained.

2. The lack of programmability [1-8, 10-15]. Although programmability can be achieved in most of the circuits by replacing the grounded resistors involved with OTAs configured as grounded resistors [21], this implies the use of additional active elements with increased power consumption and chip area.

\footnotetext{
* Corresponding author. E-mail: mtaher@kfupm.edu.sa
} 
3. The use of excessive number of resistors; three resistors or more [1-7, 11-14].

4. The lack of oscillation condition that makes it impossible to control the amplitude of oscillation [19].

5. The use of the internal parasitic of the active devices. This implies that the frequency of oscillation may not be well defined as it is a function of the parasitic of the active devices which are themselves function of the dc supply voltage and the temperature $[6,8,9,11]$.

6. High output impedance and/or frequency-dependent output impedance [6, 7, 9-20].

On the other hand, a sinusoidal oscillator can be synthesized using the parallel combination of a positive resistance, an ideal capacitance, an ideal inductance and a negative resistance. Alternatively, if all the impedances are scaled by $s / \omega$ then a sinusoidal oscillator can be synthesized using a network formed of the parallel combination of a positive inductance, a positive conductance, a frequency dependent negative conductance and a negative inductance. The advantages of realizing sinusoidal oscillators through such networks are well documented in the literature [22]. Similarly, a sinusoidal oscillator can be synthesized from the parallel combination of a positive capacitance, a positive conductance, a frequency-dependent negative conductance, and a negative capacitance. Recently a precise immittance function simulator has been presented [23]. Using a single CFOA, two capacitors and two grounded resistors, a grounded immittance simulator comprising the parallel combination of a negative inductor, a negative capacitor and a negative conductance can be obtained. It appears, therefore, that a suitable modification of this immittance function simulator [23] can yield a truly synthesized sinusoidal oscillator circuit and it is the major intention of this paper to present such circuit. The proposed circuit uses only two active elements, three grounded capacitors and one floating resistor and enjoys the following attractive features:

1. Low impedance outlet.

2. Programmable electronic tuning of the frequency of oscillation.

3. Independent control of the frequency and the condition of oscillation.

4. Use of grounded capacitors which is attractive for monolithic integration.

5. Use of two active elements only which is attractive for monolithic implementation and low power consumption.

6. Accountability of the parasitic terminal capacitances due to appearing in parallel with externally connected capacitances.

7. The use of minimum number of resistors.

\section{PROPOSED CIRCUIT}

Consider the immittance function simulator shown in Figure 1 [23]. Assuming that the terminal characteristics of the CFOA are given by $i_{y}=0, i_{z}=\left(1+\varepsilon_{i}\right) i_{x}, v_{x}=\left(1+\varepsilon_{v}\right) v_{y}$, $v_{o}=\left(1+\varepsilon_{o}\right) v_{z}$ where $\left|\varepsilon_{i}\right| \ll 1,\left|\varepsilon_{v}\right| \ll 1$ and $\left|\varepsilon_{o}\right| \ll 1$ represent the current and voltage tracking errors of the CFOA, routine analysis yields the input admittance of the circuit shown in Figure 1 [23]

$$
Y_{\text {in }}=\frac{i_{\text {in }}}{v_{\text {in }}}=-\lambda \frac{y_{1}\left(y_{2}+y_{3}\right)}{y_{2}}
$$

where $\lambda=1 /\left(1+\varepsilon_{i}\right)\left(1+\varepsilon_{v}\right)\left(1+\varepsilon_{o}\right)$ represents the current- and voltage-tracking nonidealities of the CFOA. If we choose $y_{1}=s C_{1}, y_{2}=G_{2}$ and $y_{3}=s C_{3}$, Eq. (1) reduces to

$$
Y_{\text {in }}=-s \lambda C_{1}+\frac{\omega^{2} \lambda C_{1} C_{3}}{G_{2}}
$$




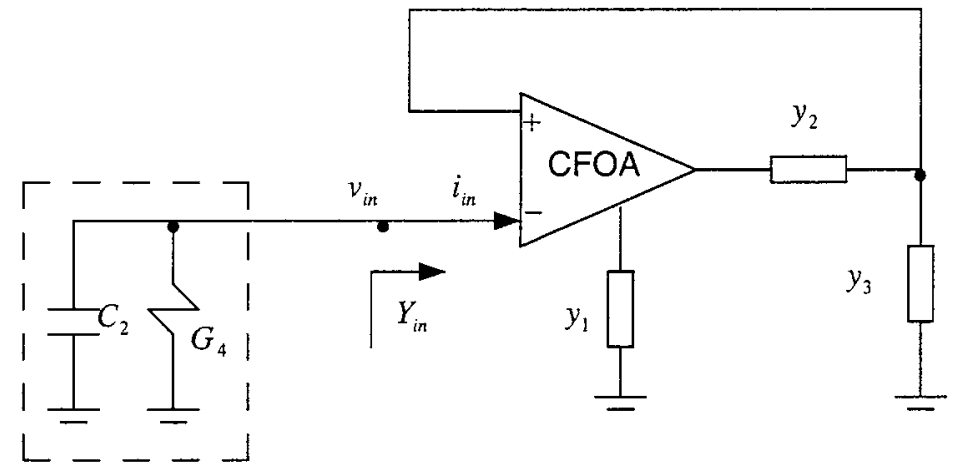

FIGURE 1 Synthesis of the oscillator from the immittance function simulator [23].

Thus, the circuit of Figure 1 can realize a grounded parallel combination of a negative capacitance $=-\lambda C_{1}$ and a frequency-dependent positive conductance $=\omega^{2} \lambda C_{1} C_{3} / G_{2}$. A sinusoidal oscillator can, therefore, be obtained from the circuit of Figure 1 by connecting a positive capacitance $C_{2}=\lambda C_{1}$ and a negative conductance $G_{4}=-\omega^{2} \lambda C_{1} C_{3} / G_{2}$ in parallel with the input port as shown, in dotted box, in Figure 1. While the condition of oscillation will be given by

$$
C_{2}=\lambda C_{1}
$$

the resulting frequency of oscillation will be given by

$$
\omega_{o}^{2}=\frac{G_{2} G_{4}}{\lambda C_{1} C_{3}}
$$

Of the many possible realizations of the negative conductance $G_{4}$, the OTA-based realization [21] was selected as it provides easy programmability by adjusting the auxiliary bias current of the OTA. The resulting oscillator circuit is shown in Figure 2. Thus, with $G_{4}=g_{m}=I_{a b c} / 2 V_{T}, g_{m}$ is the transconductance of the OTA, $I_{a b c}$ is the auxiliary bias current of the OTA and $V_{T}$ is the thermal voltage, Eq. (4) reduces to

$$
\omega_{o}^{2}=\frac{G_{2} g_{m}}{\lambda C_{1} C_{3}}
$$

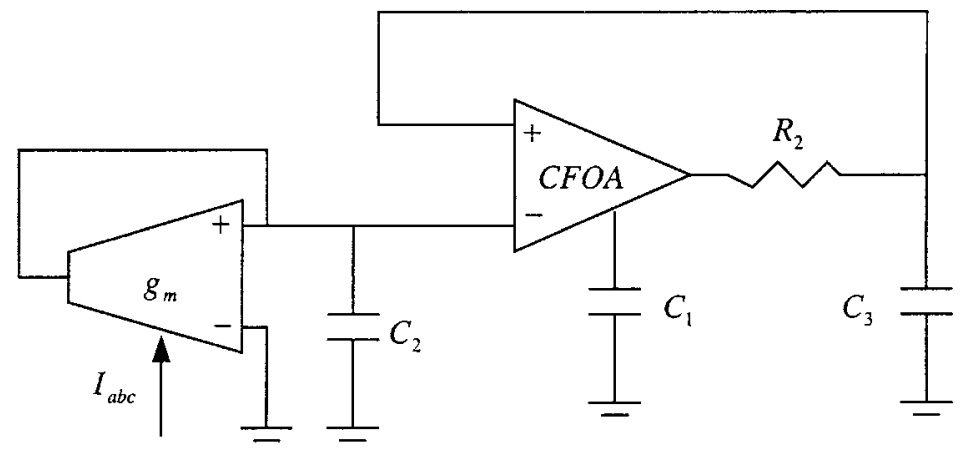

FIGURE 2 Proposed sinusoidal oscillator circuit. 
Equations (3) and (5) clearly show that while the frequency of oscillation can be controlled by adjusting $g_{m}$ without disturbing the condition of oscillation, the condition of oscillation can be controlled by adjusting the capacitance $C_{2}$ without disturbing the frequency of oscillation. Thus, the proposed oscillator circuit enjoys the attractive features of independent control of the frequency and the condition of oscillation in addition to the programmable control of the frequency of oscillation. Moreover, inspection of Eqs. (3) and (5) shows that the frequency and the condition of oscillation will be slightly affected by the current- and voltage-tracking errors of the CFOA. Furthermore, inspection of the circuit of Figure 2 shows that the parasitic capacitances on terminals $z$ and $y$ of the CFOA and the non-inverting input of the OTA can be easily absorbed by the externally connected capacitors $C_{1}, C_{2}$ and $C_{3}$.

\section{EXPERIMENTAL RESULTS}

The proposed circuit of Figure 2 was experimentally tested using the CFOA AD844, the OTA LM13600. The results obtained with different values of resistances and capacitances are shown in Figure 3. Shown also in Figure 3 are the calculated frequencies of oscillation using Eq. (5). It appears from Figure 3 that the agreement between the measured and calculated results is excellent. It must be mentioned, however, that the excellent agreement between calculated and measured results may be degraded if the amplitude of oscillation

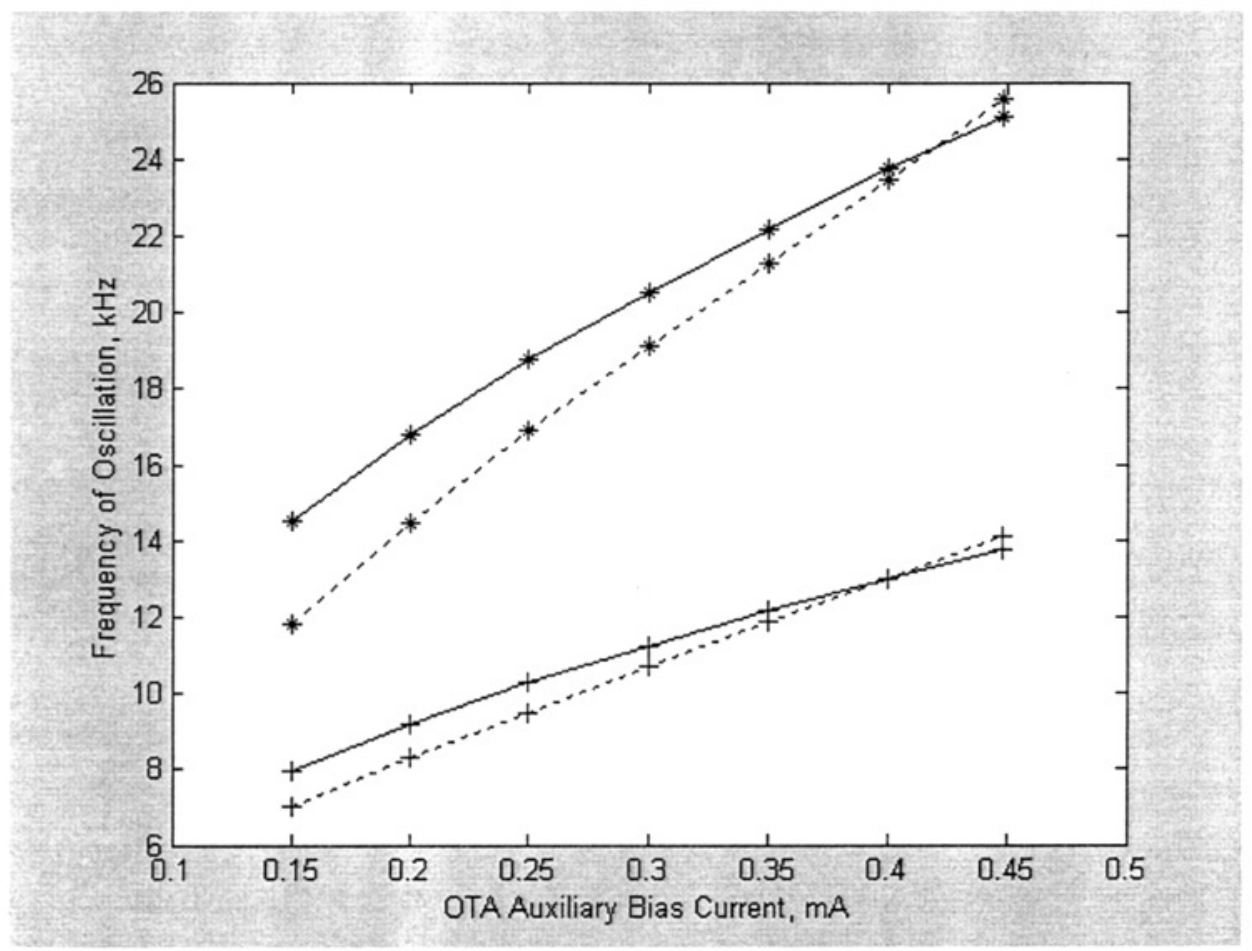

FIGURE 3 Variation of the frequency of oscillation with the OTA auxiliary bias current calculated (solid line) and measured (dotted line).

$+: R_{2}=1 / G_{2}=20 \mathrm{k} \Omega, C_{1}=1.2 \mathrm{nF}, C_{2}=1.2 \mathrm{nF}, C_{3}=50 \mathrm{nF}$

$*: R_{2}=1 / G_{2}=20 \mathrm{k} \Omega, C_{1}=1.8 \mathrm{nF}, C_{2}=1.8 \mathrm{nF}, C_{3}=10 \mathrm{nF}$. 
is large enough to drive the OTA operation into the nonlinear part of its characteristics. Obviously this can be avoided either by using limiting diodes at the input of the OTA or using an OTA with larger dynamic range than that of the LM13600.

\section{DISCUSSION AND CONCLUSION}

Using a truly synthesis approach a new sinusoidal oscillator circuit has been developed. The circuit uses two active elements, namely one OTA and one CFOA and four passive elements. When compared with previously published circuits using the same number of passive elements [16-18], the proposed circuit enjoys several attractive features. For example, the proposed circuit of Figure 3 simultaneously enjoys electronic programmability of the frequency of oscillation and can provide a low impedance outlet. The circuits of Figure 1 of Ref. [16], Figure 2 of Ref. [17] and Figure 12b,c,d of Ref. [18] use the same number of passive elements, namely four, enjoy only the electronic programmability of the frequency of oscillation and none of them can provide a low impedance outlet. In each circuit an additional active element, that is a buffer, is needed to provide low impedance outlet. Moreover, when compared with circuits using fewer number of passive components, namely three, of Figure 12a of Ref. [18] and Figure 1 of Ref. [20], again the circuits of Refs. [18] and [20] can support only electronic programmability of the frequency of oscillation, and require an additional active element, that is a buffer, for providing low impedance outlets. Furthermore, the amplitude of oscillation of the proposed circuit can be adjusted by controlling the condition of oscillation. While the circuit of Figure 1 of Ref. [19] uses only two passive elements and enjoys electronic programmability of the frequency of oscillation, it can not provide a low impedance outlet and its amplitude of oscillation can not be adjusted as it suffers from the serious disadvantage of not having a condition of oscillation. Finally, compared with similar circuits which can provide a low impedance outlet [1-5], the proposed circuit uses four passive elements and enjoys electronic programmability of the frequency of oscillation while all the other circuits [1-5] require five passive elements and do not enjoy the electronic programmability of the frequency of oscillation. Compared with the proposed circuit of Figure 3, all other circuits [6-15] suffer from one or more of the following disadvantages:

1. Require excessive number of passive components [7, 10, 13-15].

2. Rely on the parasitic of the active elements $[6,8,9,11]$.

3. Use commercially unavailable active elements; for example the minus-type firstgeneration current-conveyor $[10,12,15]$ or the minus-type second-generation currentconveyor [14].

4. Can not support low impedance outlet [7, 9-15].

5. Can not support electronic programmability of the frequency of oscillation without using additional active elements [6-8, 10-15].

It appears, therefore, that the developed circuit of Figure 3 simultaneously enjoys a number of features which do not exist in any of the previously reported circuits [1-20].

\section{References}

[1] Senani, R. and Singh, V. K. (1996). Novel single-resistance-controlled-oscillator configuration using current feedback amplifiers. IEEE Transactions on Circuits and Systems-I: Fundamental Theory and Applications, 43, 698-700.

[2] Gupta, S. S. and Senani, R. (1998). State variable synthesis of single resistance controlled grounded capacitor oscillators using only two CFOAs. IEE Proceedings - Circuits, Devices and Systems, 145, 135-138. 
[3] Gupta, S. S. and Senani, R. (1998). State variable synthesis of single resistance controlled grounded capacitor oscillators using only two CFOAs: additional new results. IEE Proceedings-Circuits, Devices and Systems, 145, 415-418.

[4] Martinez, P. A., Sabadell, J. and Aldea, C. (1997). Grounded resistor controlled sinusoidal oscillator using CFOAs. Electronics Letters, 33, 346-348.

[5] Senani, R. (1998). Realization of a class of analog signal processing/signal generation circuits: Novel configurations using current feedback op-amps. FREQUENZ, 52, 196-206.

[6] Abuelma'atti, M. T. and Al-Zaher, H. A. (1998). New grounded-capacitor sinusoidal oscillators using the current-feedback-amplifier pole. Active and Passive Electronic Components, 21, 23-32.

[7] Abuelma'atti, M. T. and Al-Zaher, H. A. (1998). Current-mode sinusoidal oscillators using two FTFNs. Proceedings National Science Council, ROC(A), 22, 758-764.

[8] Abuelma'atti, M. T. (2001). Identification of two-amplifier partially active-R sinusoidal oscillators. Proceedings National Science Council, ROC(A), 25, 127-130.

[9] Abuelma'atti, M. T. and Khan, M. H. (1996). New minimum-component OTA-based sinusoidal oscillators. Active and Passive Electronic Components, 19, 33-40.

[10] Abuelma'atti, M. T. and Al-Ghumaiz, A. A. (1996). Novel CCI-based single-element-controlled oscillators employing grounded resistors and capacitors. IEEE Transactions on Circuits and Systems-I: Fundamental Theory and Applications, 43, 153-155.

[11] Abuelma'atti, M. T. and Khan, M. H. (1997). New sinusoidal oscillators employing the CCII internal pole. International Journal of Electronics, 83, 817-823.

[12] Abuelma'atti, M. T. and Al-Ghumaiz, A. A. (1995). Novel current-conveyor-based single-element-controlled oscillator employing grounded resistors and capacitors. Active and Passive Electronic Components, 17, 203-206.

[13] Abuelma'atti, M. T. and Khan, M. H. (1995). On the realization of low/high frequency CCII+ based oscillators employing grounded resistors and lossy capacitors. Active and Passive Electronic Components, 17, 269-274.

[14] Abuelma'atti, M. T., Al-Ghumaiz, A. A. and Khan, M. H. (1995). Novel CCII- based single-element controlled oscillators employing grounded resistors and capacitors. International Journal of Electronics, 78, 1107-1112.

[15] Abuelma'atti, M. T., Al-Ali, A. A. and Ahsan, M. (1995). Programmable current-conveyor-based oscillator employing grounded resistors and capacitors. Active and Passive Electronic Components, 18, 259-265.

[16] Abuelma'atti, M. T., Al-Ali, A. A. and Lahloub, M. (1994). Self-tuned oscillator with grounded RC. Electronic Engineering, 66, 30.

[17] Abuelma'atti, M. T. (1993). New current-controlled CCII-based RC oscillators with grounded capacitors and resistors. International Journal of Circuit Theory and Applications, 21, 487-491.

[18] Tao, Y. and Fidler, J. K. (2000). Electronically tunable dual-OTA second-order sinusoidal oscillators/filters with non-interacting controls: A systematic synthesis approach. IEEE Transactions on Circuits and Systems-I: Fundamental Theory and Applications, 47, 117-129.

[19] Abuelma'atti, M. T. (1992). A new minimum component electronically tunable OTA-C sinusoidal oscillator with grounded capacitors. Modelling, Measurement \& Simulation, A, 48(3), 27-30.

[20] Abuelma'atti, M. T. (1990). A new minimum component active-C OTA-based linear voltage (current)-controlled sinusoidal oscillator. IEEE Transactions on Instrumentation and Measurement, 39, 795-797.

[21] Geiger, R. L. and Sanchez-Sinencio, E. (1985). Active filter design using operational transconductance amplifiers: a tutorial. IEEE Circuits and Devices Magazine, 1, 20-32.

[22] Senani, R. (1982). A class of single-element-controlled sinusoidal oscillators. AEU (Germany), 36, 405-408.

[23] Cicekoglu, O. (1998). Precise simulation of immittance functions using the CFOA. Microelectronics Journal, 29, 973-975. 

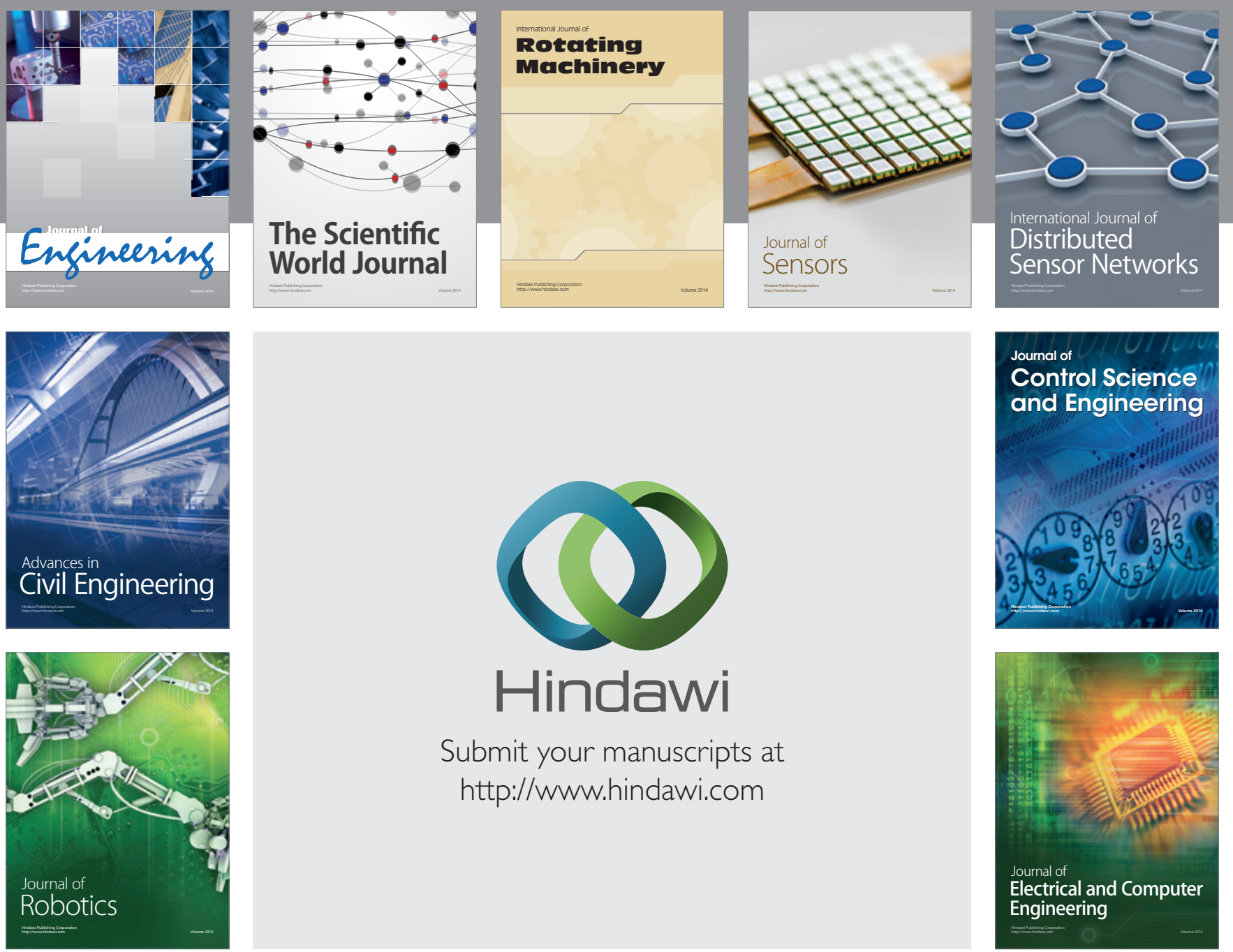

Submit your manuscripts at

http://www.hindawi.com
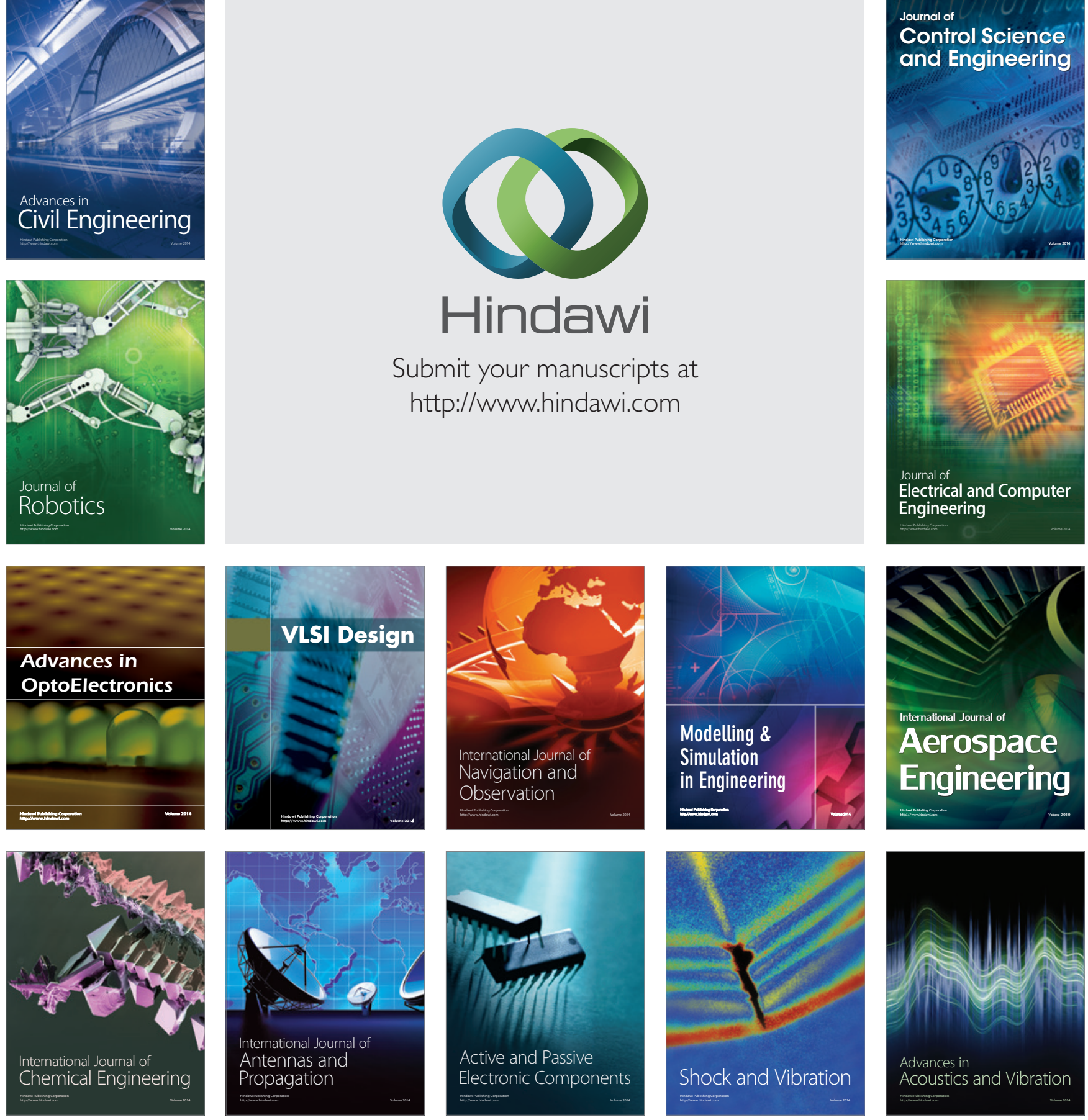